\title{
A Case Report Discussing an Uncommon Peripheral Exudative Hemorrhagic Chorioretinopathy
}

\author{
Marlon J. Demeritt \\ Nova Southeastern University, dmarlon@nova.edu \\ Beata I. Lewandowska \\ Nova Southeastern University, blewandows@nova.edu
}

Follow this and additional works at: https://nsuworks.nova.edu/ijahsp

Part of the Eye Diseases Commons

\section{Recommended Citation}

Demeritt MJ, Lewandowska BI. A Case Report Discussing an Uncommon Peripheral Exudative Hemorrhagic Chorioretinopathy. The Internet Journal of Allied Health Sciences and Practice. 2021 Jan 01;19(1), Article 1.

This Case Study is brought to you for free and open access by the College of Health Care Sciences at NSUWorks. It has been accepted for inclusion in Internet Journal of Allied Health Sciences and Practice by an authorized editor of NSUWorks. For more information, please contact nsuworks@nova.edu. 


\title{
A Case Report Discussing an Uncommon Peripheral Exudative Hemorrhagic Chorioretinopathy
}

\begin{abstract}
Purpose: The purpose of this case report is to discuss an uncommon, unique hemorrhagic chorioretinopathy. Method: A 79-year-old-white female presented to clinic with complaints of difficulty reading at close distances and seeing faces at far distances in both eyes. An 85-year old white female presented with no visual complaints but stated redness in the nasal corner of her right eye. This is a case report of two patients that presented with geographic atrophy of the macula as well as midperipheral RPE alterations in both eyes, which resembled intraretinal hemorrhages. Results: The ocular findings associated with peripheral exudative hemorrhagic chorioretinopathy may include midperipheral intraretinal hemorrhages, peripheral RPE mottling, subretinal fluid or hemorrhages, subretinal exudation, pigment epithelial detachments, and/or vitreous hemorrhages. Conclusions: Although this condition is rare, primary eye care providers should be aware of this unique clinical entity, its relationship to age-related macular degeneration, and when these patients require a referral to a retinal specialist.
\end{abstract}

\author{
Author Bio(s) \\ Marlon Demeritt, OD, MBA, FAAO is an Assistant Professor and Attending Optometrist at Nova \\ Southeastern University College of Optometry and The Eye Care Institute.
}

Beata Lewandowska, $O D$ is an Assistant Professor and Attending Optometrist at Nova Southeastern University College of Optometry and The Eye Care Institute.

\section{Acknowledgements}

1. Shields, Jerry A., et al. "Pseudomelanomas of the posterior uveal tract: the 2006 Taylor R. Smith Lecture." Retina 25.6 (2005): 767-7 2. Shields, Carol L., et al. "Peripheral exudative hemorrhagic chorioretinopathy simulating choroidal melanoma in 173 eyes." Ophthalmology 116.3 (2009): 529-535. 3. Mantel, Irmela, Ann Schalenbourg, and Leonidas Zografos. "Peripheral exudative hemorrhagic chorioretinopathy: polypoidal choroidal vasculopathy and hemodynamic modifications." American journal of ophthalmology 153.5 (2012): 910-922. 4. Annesley Jr, William H. "Peripheral exudative hemorrhagic chorioretinopathy." Transactions of the American Ophthalmological Society 78 (1980): 321. 5. Cebeci, Zafer, et al. "Clinical features and course of patients with peripheral exudative hemorrhagic chorioretinopathy." Turkish journal of ophthalmology 46.5 (2016): 215. 6. Mantel, Irmela, Sylvie Uffer, and Leonidas Zografos. "Peripheral exudative hemorrhagic chorioretinopathy: a clinical, angiographic, and histologic study." American journal of ophthalmology 148.6 (2009): 932-938. 7. Alforja, María Socorro, et al. "Intravitreal bevacizumab injection for peripheral exudative hemorrhagic chorioretinopathy." Japanese journal of ophthalmology 55.4 (2011): 425-427. 8. Pinarci, E. Y., et al. "Clinical characteristics of peripheral exudative hemorrhagic chorioretinopathy and its response to bevacizumab therapy." Eye 27.1 (2013): 111-112. 9. Reese, Algernon B., and Ira S. Jones. "Hematomas under the retinal pigment epithelium." American journal of ophthalmology 53.6 (1962): 897-910. 10. Gomi, Fumi, and Yasuo Tano. "Polypoidal choroidal vasculopathy and treatments." Current opinion in ophthalmology 19.3 (2008): 208-212. 11. Takkar, Brijesh, et al. "Peripheral choroidal neovascular membrane in a case of peripheral exudative hemorrhagic chorioretinopathy managed with combination therapy." International ophthalmology 37.2 (2017): 429-43. 


\title{
IIJAHSP \\ The Internet Joumnal of Allied Health Sciences and Practice \\ Dedicated to allied health professional practice and education
}

Vol. 19 No. 1 ISSN 1540-580X

\section{A Case Report Discussing an Uncommon Peripheral Exudative Hemorrhagic Chorioretinopathy}

\author{
Marlon J. Demeritt \\ Beata I. Lewandowska \\ Nova Southeastern University \\ United States
}

\begin{abstract}
Purpose: The purpose of this case report is to discuss an uncommon, unique hemorrhagic chorioretinopathy. Method: A 79-yearold-white female presented to clinic with complaints of difficulty reading at close distances and seeing faces at far distances in both eyes. An 85-year old white female presented with no visual complaints but stated redness in the nasal corner of her right eye. This is a case report of two patients that presented with geographic atrophy of the macula as well as midperipheral RPE alterations in both eyes, which resembled intraretinal hemorrhages. Results: The ocular findings associated with peripheral exudative hemorrhagic chorioretinopathy may include midperipheral intraretinal hemorrhages, peripheral RPE mottling, subretinal fluid or hemorrhages, subretinal exudation, pigment epithelial detachments, and/or vitreous hemorrhages. Conclusions: Although this condition is rare, primary eye care providers should be aware of this unique clinical entity, its relationship to age-related macular degeneration, and when these patients require a referral to a retinal specialist.
\end{abstract}

Keywords: subretinal hemorrhage, intraretinal hemorrhage, geographic atrophy 


\section{INTRODUCTION}

Peripheral exudative hemorrhagic chorioretinopathy (PEHCR) is an uncommon bilateral peripheral retinal degeneration that affects elderly patients. The clinical findings of PEHCR vary from peripheral retinal pigment epithelial mottling, subretinal fluid or hemorrhages, subretinal exudation, pigment epithelial detachments to vitreous hemorrhages. Several conditions have been mistaken for PEHCR, such as retinal capillary hemangioma, retinal macroaneurysm, retinal telangiectasia, choroidal detachment and choroidal melanoma, with choroidal melanoma being the most common misdiagnosis for PEHCR. In 2005, Shields et al. found that out of 12,000 patients referred with presumed uveal melanoma, $13 \%$ were found to have PEHCR. ${ }^{1}$ We report two cases of PEHCR with its unique presentation in patients that were examined in our clinic on two separate occasions.

\section{CASE REPORT \#1}

A 79-year-old-white female presented with complaints of difficulty reading at close distances and seeing faces at far distances in both eyes. She presented with best-corrected visual acuities of 20/80 OD and OS. Pupils were equal, round, and reactive to light, with no afferent pupillary defect (APD). Confrontation visual fields were full to finger counting OD, OS, and the motility was full range of motion OU. Slit lamp examination was remarkable for intraocular implants (PCIOL) OU. Dilated fundus examination (DFE) of the right eye revealed macular geographic atrophy and a blot hemorrhage 1.5 DD superior to the optic nerve. DFE of the left eye revealed macular geographic atrophy, and few dot-blot hemorrhages inferior to the optic nerve. Mid-peripheral retinal evaluation of both eyes revealed various areas of RPE changes, which resembled intra-retinal hemorrhages. Peripheral retinal evaluation of the right eye revealed a subretinal hemorrhage and a retinal scar due to a resolved subretinal hemorrhage. Peripheral retina evaluation of the left eye revealed a scar due to a resolved subretinal hemorrhage. The patient was referred to a retinal specialist for further evaluation, who confirmed the suspicion of peripheral exudative hemorrhagic chorioretinopathy (PEHCR) and non-exudative age-related macular degeneration OU and recommended observation without treatment.

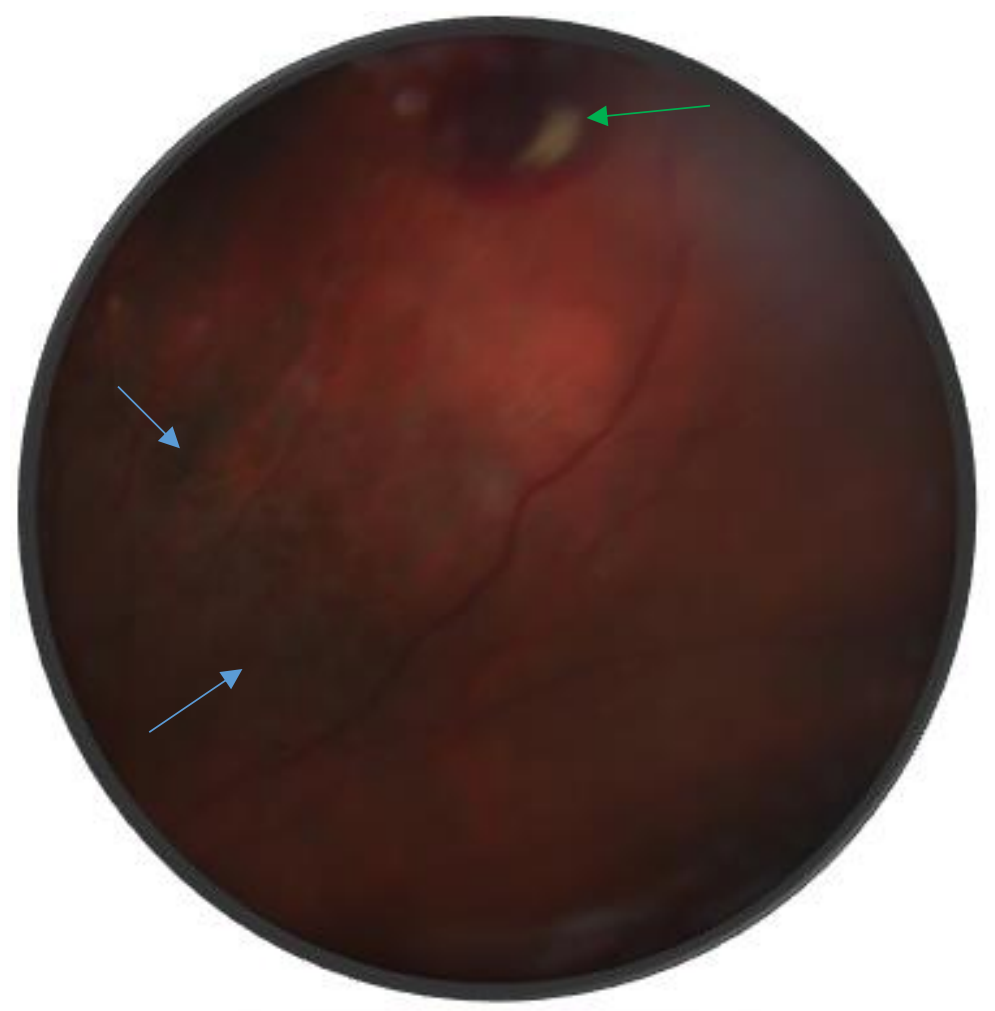

Figure 1. A digital photograph depicting multiple areas of mid-peripheral RPE alterations (blue arrows) suggestive of intraretinal hemorrhages and an isolated peripheral subretinal hemorrhage superiorly (green arrow). 


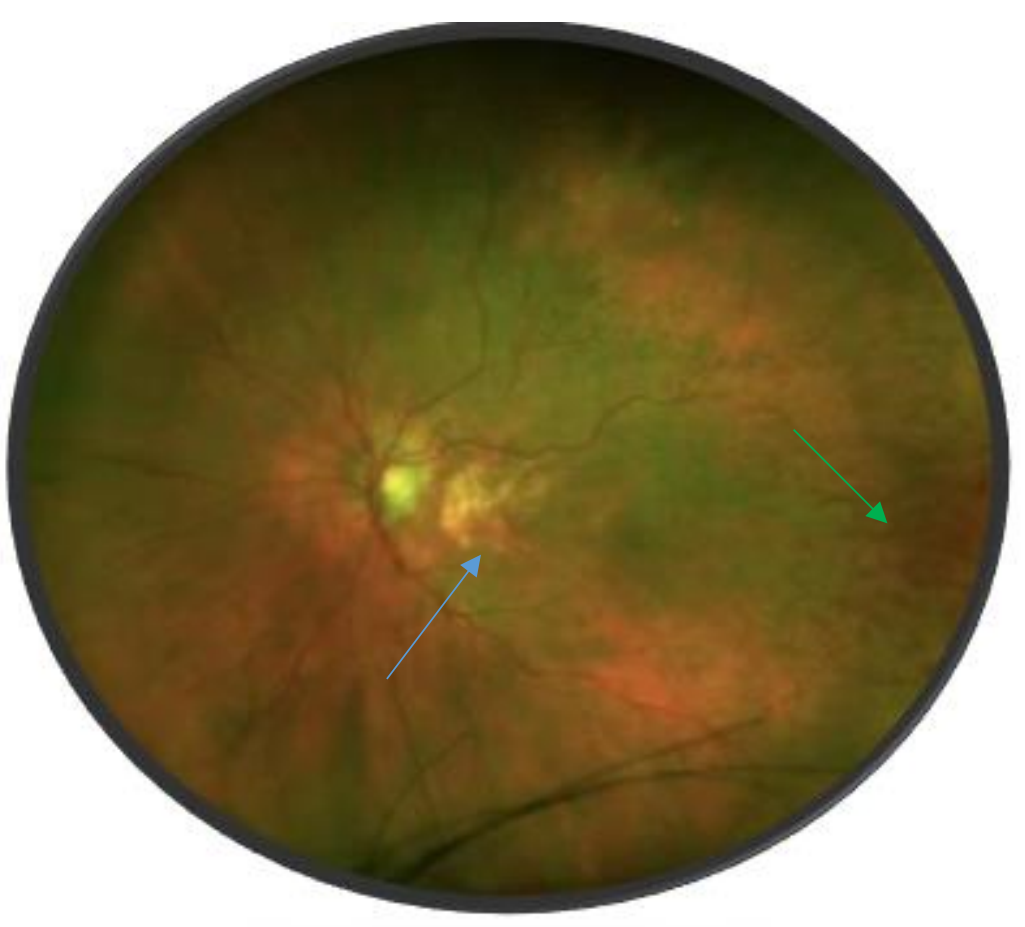

Figure 2. Wide-field fundus photograph depicting geographic atrophy (blue arrow) as well as mid-peripheral and peripheral RPE alterations (green arrow) suggestive of intraretinal hemorrhages temporally.

\section{CASE REPORT \#2}

An 85-year old white female presented with complaints of redness in the nasal corner of her right eye. Her best-corrected visual acuities were 20/70 OD and OS (she had no new visual complaints). Pupils were round and reactive to light OD, and fixed OS,

with no APD by reverse testing. Confrontation visual fields were full to finger counting OD and OS, and she demonstrated a full range of ocular motion OU. Slit lamp examination was remarkable for corneal edema OS, and PCIOL OU. DFE of the right eye and left eye revealed soft drusen and geographic atrophy OU. Mid-peripheral retinal evaluation of both eyes revealed midperipheral RPE changes, which resembled intraretinal hemorrhages. Peripheral retinal evaluation of both eyes revealed RPE pigmentary changes. The patient was referred to a retinal specialist for further evaluation, who confirmed the diagnosis of peripheral exudative hemorrhagic chorioretinopathy (PEHCR) and non-exudative age-related macular degeneration OU. Management consisted of observation alone. 


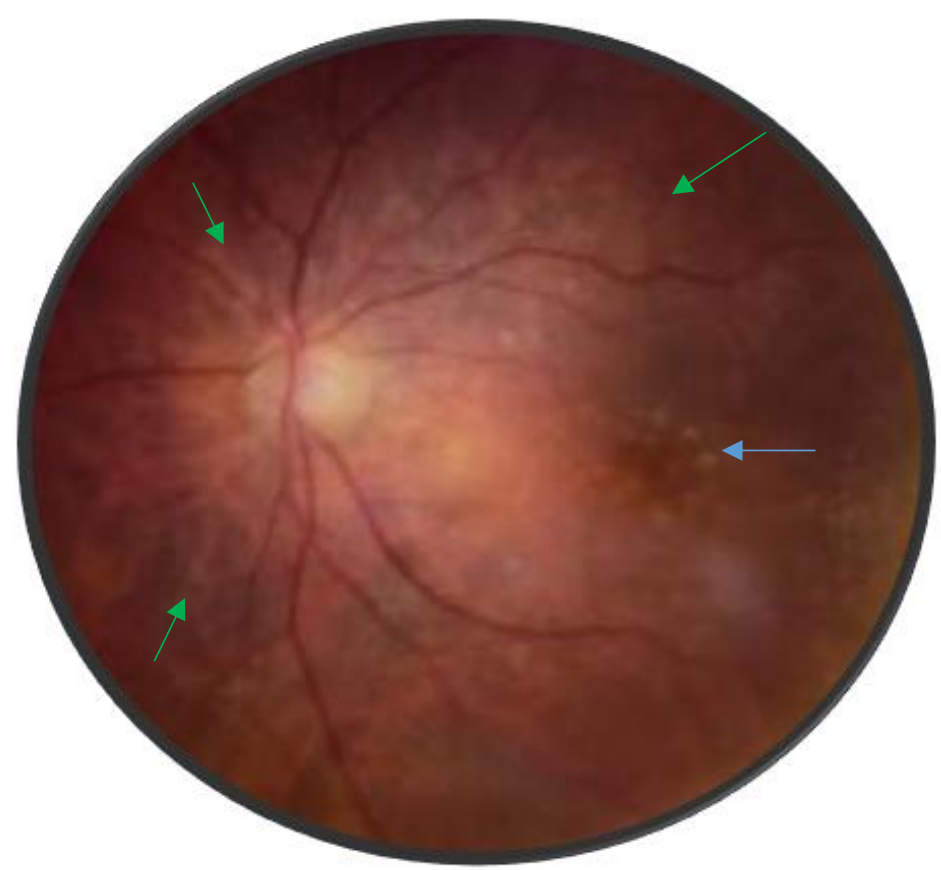

Figure 3. A digital photograph of a patient with corneal edema depicting macular drusen (blue arrow) as well as mid-peripheral RPE alterations (green arrows) suggestive of intraretinal hemorrhages superiorly, nasally, and temporally.

\section{DISCUSSION}

PEHCR is a rare degenerative disease of the peripheral retina predominantly seen in elderly women and accompanied by hemorrhage and/or exudation.1,2Advanced age serves as a risk factor for development of PEHCR. Fluctuation of systemic blood pressure and anticoagulant use are additional risk factors for hemorrhages and play a role in recurrent hemorrhages. ${ }^{3}$ The clinical features of PEHCR are described based on peripheral and macular pathology. In 2016, Cebeci et al reviewed retrospectively the records of 12 elderly patients ( 21 eyes, 4 males and 8 females) diagnosed with PEHCR. They reported that $66.7 \%$ of the patients were referred with a diagnosis of a choroidal mass. Seventy-five percent (75\%) had bilateral involvement. Subretinal hemorrhages and hemorrhagic/serous retinal pigment epithelial detachments were found in $71.4 \%$ of eyes, lipid exudation in $52.4 \%$, chronic RPE alterations in $23.8 \%$, subretinal fibrosis is $9.5 \%$ and vitreous hemorrhage in $4.8 \%$. Drusen accompanied the PEHC lesions in $52.4 \%$ of eyes, geographic atrophy was noted in $9.5 \%$, and CNV scars in $9.5 \% .^{5}$

Due to their appearance as dark, elevated masses, PEHCR is often mistaken for choroidal melanoma. In 2009, Shields et al reviewed retrospectively the records of 146 elderly patients ( 173 eyes, $67 \%$ female, $99 \%$ Caucasian). All were referred for possible choroidal melanoma. The lesions were located temporally in $77 \%$ of eye, involved 1 or 2 quadrants in $92 \%$, and were located between the equator and ora serrata in $89 \%$. Subretinal hemorrhage was found in $78 \%$ of eyes, retinal exudation in $21 \%$, RPE detachment in $28 \%$, sub-RPE hemorrhage in $26 \%$. Peripheral RPE alterations or drusen were found in $69 \%$ of ipsilateral and $42 \%$ of contralateral eyes. Macular RPE alterations, drusen, or CVM were found in $48 \%$ of ipsilateral and $56 \%$ of contralateral eyes. Lesion stability or regression was reported in $89 \%$ of eyes and progression in $11 \%$. In addition, Shields et al. highlighted clinical features that can be used to distinguish between PEHCR and choroidal masses. These include a more peripheral location of the lesions in PEHCR, absence of sentinel vessels on anterior segment, retinal exudation, macular and peripheral RPE alterations, appearance of hypofluorescence on fluorescein angiography (FA) and absence of intrinsic vascular pulsation and presence of a clot retraction cleft on ultrasonography. ${ }^{2}$

Patients can be asymptomatic, otherwise they may complain of visual loss, floaters, photopsia, metamorphopsia, and scotoma. ${ }^{3} \mathrm{PEHCR}$ lesions are commonly located in the temporal retina between the equator and the ora serrata. ${ }^{2-5} \mathrm{~V}$ Visual acuity in PEHCR can be affected due to presence of vitreous hemorrhages, macular edema and/or accompanying AMD- related findings such as drusen, choroidal neovascular membrane (CNV) and geographic atrophy. Shields et al. observed age-related macular changes in $17 \%$ of ipsi- and $27 \%$ of contralateral eyes in PEHCR patients. ${ }^{2}$ PEHCR is diagnosed by patient history, ophthalmoscopic findings and testing. Fundus fluorescein angiography findings include hypofluorescence consistent with hemorrhage in the lesion area and irregular hyperfluorescence shown by CNV and homogenous hyperfluorescence typical of serous PED in the periphery. Indocyanine green angiography can show polypoid formations and abnormal choroidal 
vasculature. ${ }^{4}$ Usually, PECHR remains stable or regresses spontaneously, which can result in atrophy, fibrosis, or hyperplasia. ${ }^{5}$ The treatment options for PEHCR include the following: monitoring, vitrectomy, intravitreal anti-VEGF therapy, cryotherapy, laser photocoagulation, or photodynamic therapy (PDT).6,7

The prognosis of PEHCR depends on the location of the lesion. In cases where the central vision or macula remain unaffected, observation without intervention is recommended as the disease may spontaneously regress. ${ }^{8}$ Laser photocoagulation or PDT are reserved for cases that are threatening the macula and for peripheral polyps. ${ }^{9} \mathrm{~A}$ pars plana vitrectomy is recommended in rare cases where vitreous hemorrhage or massive subretinal hemorrhage are present. ${ }^{10}$ Choroidal neovascular membranes in the periphery generally have a good prognosis, because they can be safely treated with laser therapy without the risk of central vision loss. ${ }^{11}$

\section{Conclusion}

PEHCR is an uncommon, peripheral retinal degeneration that primarily affects elderly patients. Ocular findings may include midperipheral RPE alterations, which resemble intraretinal hemorrhages, peripheral RPE mottling, subretinal fluid or hemorrhages, subretinal exudation, pigment epithelial detachments, and/or vitreous hemorrhages. Although this condition is rare, primary eye care providers should be aware of this unique clinical entity, its relationship to age-related macular degeneration, and that if there is macular involvement or a rapidly progressive lesion, a referral to a retinal specialist is warranted.

A part of this manuscript has been used in a poster presentation at the 2018 and 2019 SECO Congress.

\section{References}

1. Shields, Jerry A., et al. "Pseudomelanomas of the posterior uveal tract: the 2006 Taylor R. Smith Lecture." Retina 25.6 (2005): 767-7

2. Shields, Carol L., et al. "Peripheral exudative hemorrhagic chorioretinopathy simulating choroidal melanoma in 173 eyes." Ophthalmology 116.3 (2009): 529-535.

3. Mantel, Irmela, Ann Schalenbourg, and Leonidas Zografos. "Peripheral exudative hemorrhagic chorioretinopathy: polypoidal choroidal vasculopathy and hemodynamic modifications." American journal of ophthalmology 153.5 (2012): 910-922.

4. Annesley Jr, William H. "Peripheral exudative hemorrhagic chorioretinopathy." Transactions of the American Ophthalmological Society 78 (1980): 321.

5. Cebeci, Zafer, et al. "Clinical features and course of patients with peripheral exudative hemorrhagic chorioretinopathy." Turkish journal of ophthalmology 46.5 (2016): 215.

6. Mantel, Irmela, Sylvie Uffer, and Leonidas Zografos. "Peripheral exudative hemorrhagic chorioretinopathy: a clinical, angiographic, and histologic study." American journal of ophthalmology 148.6 (2009): 932-938.

7. Alforja, María Socorro, et al. "Intravitreal bevacizumab injection for peripheral exudative hemorrhagic chorioretinopathy." Japanese journal of ophthalmology 55.4 (2011): 425-427.

8. Pinarci, E. Y., et al. "Clinical characteristics of peripheral exudative hemorrhagic chorioretinopathy and its response to bevacizumab therapy." Eye 27.1 (2013): 111-112.

9. Reese, Algernon B., and Ira S. Jones. "Hematomas under the retinal pigment epithelium." American journal of ophthalmology 53.6 (1962): 897-910.

10. Gomi, Fumi, and Yasuo Tano. "Polypoidal choroidal vasculopathy and treatments." Current opinion in ophthalmology 19.3 (2008): 208-212.

11. Takkar, Brijesh, et al. "Peripheral choroidal neovascular membrane in a case of peripheral exudative hemorrhagic chorioretinopathy managed with combination therapy." International ophthalmology 37.2 (2017): 429-43. 